\title{
Fbmc System With Low Density Parity Check (Ldpc) Coding For Efficient Communications In
} $5 \mathrm{~g}$

\author{
Ravi Sekhar Yarrabothu, Usha Rani Nelakuditi
}

\begin{abstract}
Channel coding is a complicated subject, but vital in cellular communications, which is used for detection and correction of the bit errors caused by various types of noises, interferences. In 3rd generation and 4th Generation (3G and 4G) cellular communications, Turbo codes are predominantly used and currently 3GPP standardization group considering the Low Density Parity Check (LDPC) code in $5 G$, due to its requirements of 20GB throughput, ultra low latencies and massive Internet of Things. In 5G Communication Filter Bank Multi Carrier (FBMC) wave form is considered for $5 G$ and beyond communication for non-orthogonal communications due to its superior side-lobe suppression characteristics and also no need to use the cyclic prefix, which will save the bandwidth. With FBMC based systems, the Bit Error Rate (BER) performance is still not able to reach the LTE level, which uses Orthogonal Frequency Division Multiplexing (OFDM). In this paper, to achieve a better $B E R$ performance, it is proposed to use the LDPC coding with FBMC waveform for $5 G$ communication and was simulated with Keysight SystemVue Software. The BER analysis is performed for both coded and uncoded data transmission for FBMC system with three different modulations: QPSK, 16QAM, 64QAM. The simulated results shows that the LDPC coding with 1/2 code rate is better than other code rates $2 / 3,3 / 4$ and 5/6.
\end{abstract}

Keywords: BER, FBMC, LDPC Codes, Polar codes, OQAM

\section{INTRODUCTION}

In a typical cellular system, the received data always differ with the transmitted due to the communication errors, which are introduced by the channel conditions like multipath fading, noise and co-channel interference. To correct these errors the channel coding is used in the cellular communication systems. The turbo codes [1] are getting used as the primary channel coding technique for the $3 \mathrm{G}$ and 4G cellular standards of Third Generation Partnership Project(3GPP) [2], [3], due to its outstanding error correction ability and flexibility. Although, the Turbo codes are sufficient for the 4G, 3GPP standardization body is presently considering other than the turbo codes for $5 \mathrm{G}$ with the Low Density Parity Check (LDPC) code [4],[5], which is a well proven in WiFi, WiMAX, Ethernet and DVB-S2 standards [6]-[9].

The $5 \mathrm{G}$ requirements such as peak throughput of $20 \mathrm{Gbps}$, ultra low latency use cases and massive IoT devices

Revised Manuscript Received on August 14, 2019.

Ravi Sekhar Yarrabothu, Vignan's Foundation for Science, Research and Technology, Vadlamudi, Guntur, A.P, India(email Id: ykravi@gmail.com)

Usha Rani Nelakuditi, Vignan's Foundation for Science, Research and Technology, Vadlamudi, Guntur, A.P India (email Id: usharani.nsai@gmail.com) connectivity needs an efficient communication coding technique. These requirements of $5 \mathrm{G}$ can be accomplished by flexible turbo codes [10], with much better hardware and energy efficiencies rather than by using flexible LDPC codes. However, with the inflexible LDPC coding, it can be achieve the with much superior hardware and energy efficiencies. A hybrid approach of Turbo/LDPC coding [11] for $5 \mathrm{G}$ is beneficial, in which flexible turbo codes are used for most of the use-cases and using inflexible LDPC codes for ultra high speed and low latency use cases.

In this paper, for $5 \mathrm{G}$ system based on FBMC is used since FBMC provides a better spectral efficiency and suitability for asynchronous communication [12]. This paper is organised in to four sections. II section explains the need for LDPC for 5G requirements and III section discuss the FBMC system design with LDPC code. IV section presents and analyses the results and finally section $\mathrm{V}$ conclusions are discussed.

\section{LDPC CODES}

The 5G New Radio (NR) access technology will make a landmark change in channel coding for 3GPP cellular technologies. Turbo codes, have played a vital role as a key coding scheme in the $3 \mathrm{G}$ and $4 \mathrm{G}$ cellular communications and is going to be replaced with the LDPC codes. The key driver for this evolution is the high throughput requirement $(20 \mathrm{~Gb} / \mathrm{s})$ for $5 \mathrm{G}$ systems [13]. Both the Turbo and LDPC codes operate in a similar mechanism as the decoders in both are of message passing type where the information is propagated inside a graphical structure, which represents a code. The major distinction of LDPC codes are inherently parallel structured where as turbo codes are inherently serial structured. Hence the computation complexity for LDPC codes can be reduced by implementing greater parallelism in hardware implementations [14].

An ultra low latency of $0.5 \mathrm{~ms}$ in end-to-end system has to be achieved for the $5 \mathrm{G} \mathrm{NR}$, which is 20 times lower than the $10 \mathrm{~ms}$ achieved in 4G. Achieving an end-to-end latency of $0.5 \mathrm{~ms}$ implies a physical layer latency of 50 ?s [15], in which channel decoder must contribute a major share of this latency budget as many other physical layer components require higher latencies not possible to reduce.

The crucial factors for the selection of the Forward Error

Published By:
Blue Eyes Intelligence Engineering

\& Sciences Publication 


\section{FBMC SYSTEM WITH LOW DENSITY PARITY CHECK (LDPC) CODING FOR EFFICIENT COMMUNICATIONS IN 5G}

Correction (FEC) codes in communication systems are complexity in terms of area and power and its coding gain. In $5 \mathrm{G}$ communications, the selection of the channel coding scheme is based on its performance in terms of throughput(should be ultra high), latency (ultra low 0.5 milliseconds), good error correction capability, implementation complexity and the flexibility for various types of use cases. Keeping in view of these requirements, 3GPP standards body have adopted LDPC codes for data channels and polar codes for control channels in the $5 \mathrm{G}$ New Radio. In this paper an attempt is made to implement a $5 \mathrm{G}$ system with LDPC codes and FBMC as a waveform.

\section{FBMC BASED 5G SYSTEM WITH LDPC CODES}

FBMC based 5G systems are considered by 3GPP as well as other researchers as one of the potential technique for its superior spectral efficiency and support asynchronous communication [16]-[18]. In FBMC system, at both the transmitter and receiver the poly phase network (PPN) based prototype filters are used, since it provides lesser computational complexity. In this paper, FBMC system also uses offset quadrature amplitude modulation (OQAM) method is used for ICI, ISI elimination [19]. The basic block diagram of PPN FBMC transceiver system is shown in figure. 1

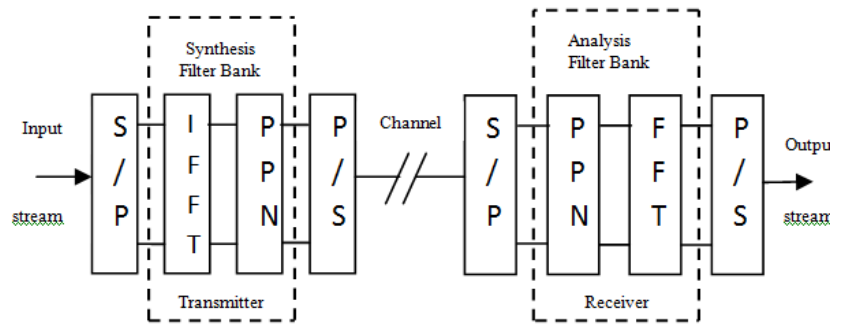

Figure 1. Block diagram of PPN-FBMC

The BER performance of the FBMC is more or less same as the OFDM systems [20] and to improve the BER performance, LDPC are to be used, so that the bandwidth efficiency can be improved. In this paper, LDPC encoding and decoding are chosen to be same as those in WLAN $11 \mathrm{ac}$ library. The code rate can only be selected from 4 options: $1 / 2,2 / 3,3 / 4,5 / 6$. The coded length can be selected from 3 options: 1944, 1296, 648. However, in this paper only code length of 1944 is tested as we are interested in higher throughputs.

The simulation of the FBMC system with LDPC code as shown in the figure 2 and is done by using Keysight SystemVue 2018 software.The frame of FBMC is composed of preamble symbols and data symbols. The preamble consists of two superimposed ZC (Zadoff-Chu) sequence. Time \& Frequency synchronization, channel estimation and equalization are done at the receiver

\section{SIMULATION RESULTS}

The simulated schemas were executed using SystemVue software (2018 version). The parameters that are set for the simulations in this paper are as given in table 2 .

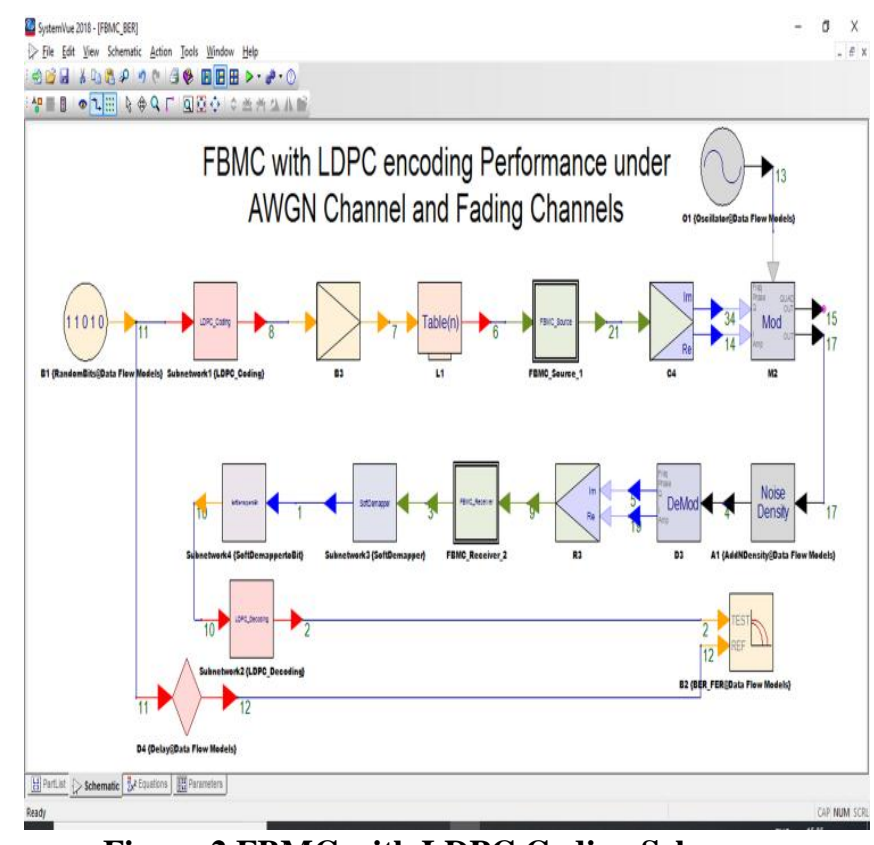

Figure 2.FBMC with LDPC Coding Schema

The BER analysis of FBMC system with various LDPC coding rates of 1/2, 2/3,3/4,5/6 and a coding length of 1944 bits under AWGN channel conditions. And also a comparison is performed with and without LDPC coding along various modulation techniques QPSK, 16QAM and 64QAM and are shown in the figures 3, 4 and 5.

Table 1. Simulation Parameters

\begin{tabular}{|l|l|}
\hline \multicolumn{1}{|c|}{ Parameter } & \multicolumn{1}{c|}{ Value } \\
\hline $\begin{array}{l}\text { Number of sub-carriers per } \\
\text { symbol }\end{array}$ & 128 \\
\hline $\begin{array}{l}\text { Number of data symbols per } \\
\text { frame }\end{array}$ & 20 \\
\hline Type of modulation & OQAM \\
\hline Channel Type & AWGN \\
\hline Spreading factore & 4 \\
\hline Filter bank structure & PPN-IFFT \\
\hline $\begin{array}{l}\text { Number of preamble symbols } \\
\text { in a frame }\end{array}$ & 6 \\
\hline LDPC Coding Rates & $1 / 2,2 / 3,3 / 4,5 / 6$ \\
\hline LDPC Code Length & 1944 bits \\
\hline
\end{tabular}

From figure 3, it can be observed that to achieve a BER of 10-4 for an uncoded data with QPSK modulation, it has to maintain an $\mathrm{Eb} / \mathrm{No}$ value of $8.5 \mathrm{~dB}$, where as for LDPC coded data with a rate of $1 / 2$ can maintain an $\mathrm{Eb} / \mathrm{No}$ value of $2.2 \mathrm{~dB}$. This shows a gain of $6.2 \mathrm{~dB}$ due to LDPC coding rate of $1 / 2$. One interesting aspect is for achieving the BER rates of 10-5 and 10-6, the coding gains are $7.2 \mathrm{~dB}$ and 8.0 $\mathrm{dB}$ as shown in the figure 3, which is better than compared to the higher BER values. 


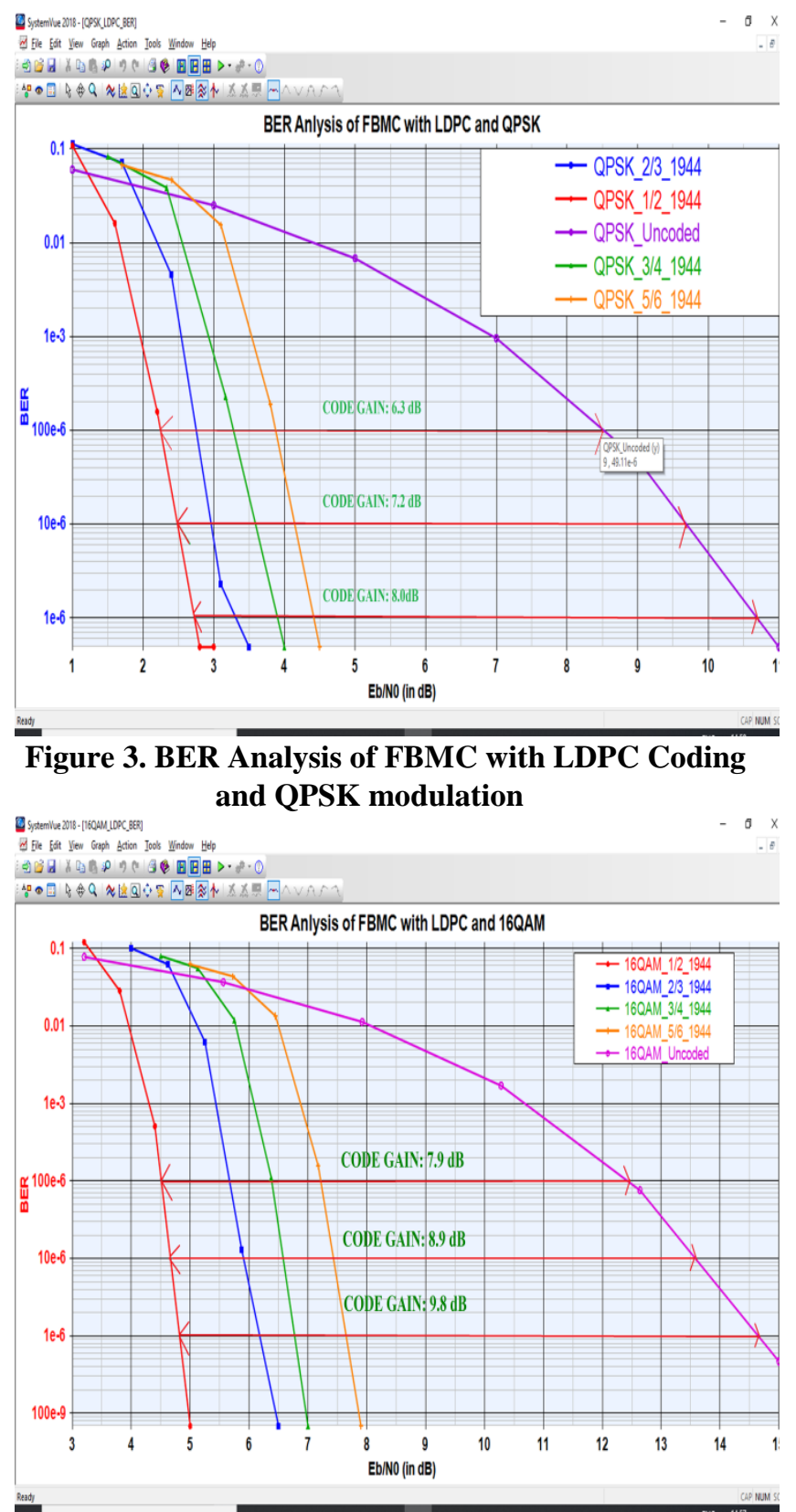

Figure 4. BER Analysis of FBMC with LDPC Coding and 16QAM modulation

From figure 4 , it can be observed that to achieve a BER of 10-4 for an uncoded data with 16QAM modulation, it has to maintain an Eb/No value of $12.4 \mathrm{~dB}$, where as for $\mathrm{LDPC}$ coded data with a rate of $1 / 2$ can maintain an Eb/No value of $4.5 \mathrm{~dB}$. This shows a gain of $7.9 \mathrm{~dB}$ due to LDPC coding rate of $1 / 2$. One interesting aspect is for achieving the BER rates of 10-5 and 10-6, the coding gains are $8.9 \mathrm{~dB}$ and 9.8 $\mathrm{dB}$ as shown in the figure 4 , which is better than compared to the higher BER values.

From figure 5, it can be observed that to achieve a BER of 10-4 for an uncoded data with QPSK modulation, it has to maintain an $\mathrm{Eb} / \mathrm{No}$ value of $16.7 \mathrm{~dB}$, where as for $\mathrm{LDPC}$ coded data with a rate of $1 / 2$ can maintain an $\mathrm{Eb} / \mathrm{No}$ value of $7.3 \mathrm{~dB}$. This shows a gain of $9.4 \mathrm{~dB}$ due to LDPC coding rate of $1 / 2$. One interesting aspect is for achieving the BER rates of 10-5 and 10-6, the coding gains are $10.4 \mathrm{~dB}$ and $11.4 \mathrm{~dB}$ as shown in the figure 5, which is better than compared to the higher BER values.

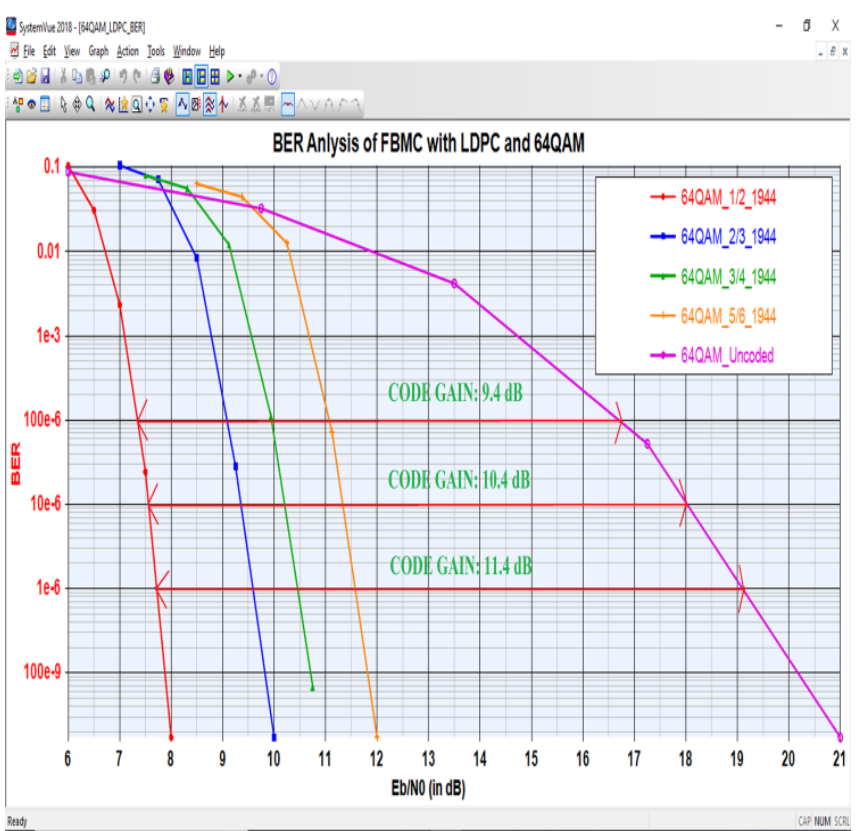

\section{Figure 5. BER Analysis of FBMC with LDPC Coding and 64QAM modulation}

One more observation from figures 3,4 and 5 is the coding gain increases as the order of QAM modulation increases and also coding rate of $1 / 2$ gives a better performance when compared to the other coding rates.

\section{CONCLUSION}

In this paper The FBMC system with LDPC coding is simulated and performance is evaluated for various modulation techniques and also with various coding rates $1 / 2,2 / 3,3 / 4,5 / 6$ and a fixed coding length of 1944 bits under AWGN channel conditions. It has been concluded that the LDPC codes provide a very good coding gain when compared with un-coded data and also the coding gain increases gradually as order of QAM modulation increases. And also as we achieve better BER performance the coding gain also higher. This work can be extended to perform the BER analysis for multipath fading conditions, so that the real time cellular conditions can be tested.

\section{ACKNOWLEDGMENT}

We would like to acknowledge the Vignaan's Foundation for Science Technology and Research and Keysight Technologies, who provided the valuable resources through the Centre of Excellence (Keysight - Vignan's Advanced RF Microwave and Wireless Communications). This project used the design tool SystemVue 2018 extensively for the simulations. We would like to express our sincere thanks and gratitude to the support engineers of the Keysight Technologies for their continual technical support during this project. 


\section{FBMC SYSTEM WITH LOW DENSITY PARITY CHECK (LDPC) CODING FOR EFFICIENT COMMUNICATIONS IN 5G}

\section{REFERENCES}

1. C. Berrou, A. Glavieux, and P. Thitimajshima, "Near Shannon limit error-correcting coding and decoding: Turbo-codes (1)," in Proc. IEEE Int. Conf. on Communications, vol. 2, Geneva, Switzerland, may 1993, pp. 1064-1070, 1993.

2. ETSI TS 125 222; Universal Mobile Telecommunications System (UMTS); Multiplexing and Channel Coding (FDD), V9.3.0 ed., 2012.

3. ETSI TS 136212 LTE; Evolved Universal Terrestrial Radio Access (E-UTRA); Multiplexing and Channel Coding, V13.1.0 ed., 2016.

4. T. Richardson and S. Kudekar, "Design of Low-Density Parity Check Codes for 5G New Radio," in IEEE Communications Magazine, vol. 56, no. 3, pp. 28-34, March 2018.

5. D. J. C. MacKay and R. M. Neal, "Near Shannon limit performance of low density parity check codes," Electron. Lett., vol. 32, no. 18, pp. 457-458, aug 1996.

6. IEEE 802.11-2012 Standard for Information Technology - Telecommunications and Information Exchange between Systems - Local and Metropolitan Area Networks - Specific Requirements - Part 11: Wireless LAN Medium Access Control (MAC) and Physical Layer (PHY), 2012.

7. IEEE 802.16-2012 Standard for Local and Metropolitan Area Networks - Part 16: Air Interface for Broadband Wireless Access Systems, 2012.

8. IEEE 802.3an-2006 Specific requirements Part 3: Carrier Sense Multiple Access with Collision Detection (CSMA/CD) Access Method and Physical Layer Specifications Amendment 1: Physical Layer and Management Parameters for $10 \mathrm{~Gb} / \mathrm{s}$ Operation, 2006.

9. ETSI EN 302 307-1 Digital Video Broadcasting (DVB); Second generation framing structure, channel coding and modulation systems for Broadcasting, Interactive Services, News Gathering and other broadband satellite applications; Part 1: DVB-S2, V1.4.1 ed., 2014.

10. R. G. Maunder, "The 5G channel code contenders," AccelerComm White Paper, Aug. 2016. [Online]. Available: http://www.accelercomm.com

11. R.G. Maunder, "A Vision for 5G Channel Coding," AccelerComm White Paper, Sept. 2016. [Online]. Available: https://eprints.soton.ac.uk/id/eprint/401809

12. RS Yarrabothu, S Kandukuri, UR Nelakuditi, MKC Rao , "Performance analysis of a 5G non-orthogonal waveform filter bank multi carrier", Electronic Government, an International Journal, vol. 13, no. 4, pp. 290-305, 2017

13. 3GPP TSG RAN Meeting \#71, RP-160671, "New SID Proposal: Study on New Radio Access Technology," NTT DOCOMO Inc., Goteborg, Sweden, 7-10 Mar. 2016.

14. F. Khan, "LTE for $4 \mathrm{G}$ Mobile Broadband: Air Interface Technologies and Performance, " Cambridge University Press, 2009.

15. G. P. Fettweis, "The Tactile Internet - applications and challenges," IEEE Veh. Technol. Mag., pp. 64-70, Mar. 2014.

16. M. Bellanger, FBMC physical layer: a primer, PHYDYAS, Jan 2010

17. P. Siohan, C. Siclet, and N. Lacaille, "Analysis and design of OFDM/OQAM systems based on filterbank theory," IEEE Transactions on Signal Processing, vol. 50, no. 5, pp. 1170-1183, 2002.

18. Van Eeckhaute, M., Bourdoux, A., De Doncker, P. Fran_cois Horlin, "Performance of emerging multicarrier waveforms for $5 \mathrm{G}$ asynchronous communications", EURASIP Journal on Wireless Communications and Networking, 2017. . [Online]. Available: https://doi.org/10.1186/s13638-017-0812-8.

19. G. Wunder et al., "5GNOW: Non-orthogonal, asynchronous waveforms for future mobile applications," IEEE Commun. Mag., vol. 52, issue. 2, pp. 97-105, Feb. 2014

20. M. Shaik and R. S. Yarrabothu, "Comparative Study of FBMC-OQAM and OFDM Communication System," 2018 2nd International Conference on Trends in Electronics and Informatics (ICOEI), Tirunelveli, India, pp. 559-563, 2018.

\section{AUTHORS PROFILE}

Ravi Sekhar Yarrabothu Vignan's Foundation for Science, Research and Technology, Vadlamudi, India

Email Id: ykravi@gmail.com

Usha Rani Nelakuditi Vignan's Foundation for Science, Research and Technology, Vadlamudi, India

Email Id: usharani.nsai@gmail.com 